\title{
Systemic Lupus Erythematosus (SLE) - perspectives for a population — based registry in São Paulo city, Brazil
}

Mortality and morbidity registries are fundamental in health research and in health services administration.

There are good mortality registries in São Paulo. However morbidity registries are few. For infectious diseases the Epidemiologic Surveillance System presents very reliable data.

Regarding chronic diseases only the cancer registries of some big cities are population-based, an example is the "Registro de Cancer" based at "Faculdade de Saúde Pública USP". It seeks for cases in diagnostic and treating centers are recieves reports from doctors as well. The registry's human resources are a doctor, a statistician and two secretaries that receive, consolidate and analyse data. The registry has had dificulties, mainly because of important human resources lack.

It would be important to establish rheumatic diseases data banks due to their high disabilties rate; scarce aethiologic knowledge and the existence of effective control treatents that can prevent complicatio.

SLE fulfils the criteria above in a special way. The following reasons justify and reinforce a population based registry or SLE in São Paulo city:

1. Existence of clear diagnosis criteria - The American Rhematology Association criteria (ARA criteria) accepted internationaly by all rheumatology services.

2. Only a few public medical centers concentrate the diagnoses and treatment of almost all cases in São Paulo and have high technical level comparable to developed countries centers, namely "Hospital das Clínicas da Faculdade de Medicina da Universidade de São Paulo (HC-FMUSP), Santa Casa de São Paulo (SC-SP), Hospital São Paulo da Escola Paulista de Medicina (EPM), Hospital do Servidor Público Estadual (HSPE), Hospital do Servidor Público Municipal (HSPM), Hospital Heliópolis".

3. Existence of a Rheumatology Association congragating most of the rheumatologists.

4. Existence of an active patients association.

Bearing those aspects in mind, a meeting of representatives of Department of Rheumatology (HC-FMUSP), Department of Preventive Medicine (FMUSP) and Department of Social Medicine (SC-SP) decided to test a case Registry System to run at outpatients service of HC-FMUSP, collecting the data bellow from medical records and computer information system:

A - Name, age, date of birth, sex, race, place, of birth, schooling level, occupation and adress.

$\mathrm{B}-$ Criteria of the patient.

C - Date of the last visit to Hospital.

4. Present situation of the patient: alive, dead(cause), kidney failure, asociated rheumatic disease(s).

The sample has 600 cases and 100 patients will have their identification data checked by a doctor. According to our experience identification data of race, schooling level and occupation do not have the expected reliability.

The data have been loaded in a EPI-INFO data bank. 